
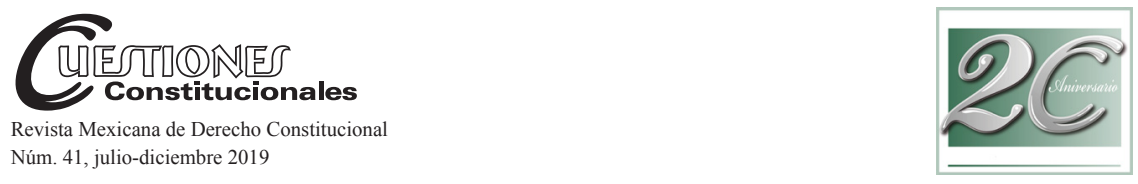

\title{
Da Tocqueville a Marshall, passando per Kelsen: il sincretismo del sistema messicano di giustizia costituzionale
}

\section{Paolo PASSAGLiA*}

\begin{abstract}
SOMMARIO: I. Introduzione. II. Da Tocqueville... (il juicio de amparo). III. ... a Marshall (l'introduzione di un controllo di costituzionalità diffuso). IV. ...passando per Kelsen (l'esistenza di un controllo astratto di costituzionalità). V. L'ibridazione come risultato di un sincretismo: la via messicana al controllo di costituzionalità.
\end{abstract}

\section{INTRODUZIONE}

La scienza comparatistica associa, generalmente, il sistema di giustizia costituzionale messicano con il juicio de amparo. Questa via d'accesso, in effetti, è una creazione assai peculiare della cultura giuridica messicana ottocentesca, che è riuscita ad imporsi, progressivamente, come un modello per molti altri sistemi del Centro e del Sudamerica. ${ }^{1}$ La sua cir-

* Professore ordinario di Diritto comparato, Università di Pisa, Italia. Coordinatore pro tempore dell'Area di diritto comparato del Servizio Studi, Corte costituzionale della Repubblica Italiana; paolo.passaglia@unipi.it.

1 Sul tema, v. Tschentscher, Axel e Lehner, Caroline, "The Latin American Model of Constitutional Jurisdiction: Amparo and Judicial Review", Social Science Research Network [SSRN], Research Paper No. 2296004, 2013, pp. 1-30, disponibile on line alla pagina http://www.servat.unibe.ch/jurisprudentia/lit/Amparo_SSRN.pdf(ultimo accesso: 12 agosto 2018 ). 
colazione è stata tanto ampia e la sua reputazione tanto elevata che, ad esempio, quando l'Assemblea costituente spagnola si trovò a strutturare un ricorso diretto individuale all'organo di giustizia costituzionale basato essenzialmente sull'istituto tedesco della Verfassungsbeschwerde, lo denominò «recurso de amparo», nonostante il prodotto finale trasfuso nella Costituzione del 1978 avesse, in concreto, ben poco a che vedere con il prototipo messicano.

L'impatto dell'amparo sulla teoria e la prassi della giustizia costituzionale è stato così potente che, in Italia, una recente tassonomia dei sistemi di giustizia costituzionale si è fondata sull'identificazione di tre modelli: quello nordamericano, quello europeo (o kelseniano) e quello basato sul giudizio di amparo messicano. ${ }^{2}$

A prescindere dalla validità euristica di una tale tassonomia, che combina due sistemi complessivamente intesi ed una specifica forma di ricorso, l'idea stessa che il giudizio di amparo non possa essere trasfuso ne (e quindi confuso con) il modello nordamericano né tantomeno in quello kelseniano merita di essere sottolineata, giacché dimostra chiaramente l'originalità della creazione messicana. L'enfasi sull'amparo, tuttavia, non dovrebbe condurre a propugnare una identificazione completa del sistema messicano di giustizia costituzionale con la sua via d'accesso più rinomata al controllo di costituzionalità. Il Messico, infatti, ha una lunga storia in tema di giustizia costituzionale, di talché il concentrare l'attenzione sul solo amparo significherebbe trascurare la sua grande complessità e la sua ricchezza, che hanno conosciuto, tra l'altro, un incremento assai rilevante nei decenni più recenti.

Obiettivo di queste pagine è tratteggiare, sia pure molto sommariamente, i termini della suddetta complessità, da un punto di vista storico, oltre che comparatistico, onde porre in rilievo le particolarità del sistema messicano di giustizia costituzionale e le forme che nel sistema sono stata elaborate per consentire la coesistenza di diverse fonti di ispirazione. Più in particolare, sono tre le fonti che possono essere individuate, e che saranno di seguito analizzate partitamente: in primo luogo, la fonte «tradizionale» che è stata alla base dell'elaborazione del juicio de amparo, e poi le due fonti più recenti, sulla scorta delle quali si è venuto a formare un sistema che è, ad

2 Cfr. E. Palici di Suni, "Tre modelli di giustizia costituzionale", Rivista AIC, 11 marzo 2016, No. 1/2016, consultabile on line alla pagina http://www.rivistaaic.it/tre-modellidi-giustizia-costituzionale.html (ultimo accesso: 12 agosto 2018), pp. 1-24, spec. p. 8. 
un tempo, accentrato ${ }^{3}$ e diffuso. Il risultato di queste diverse influenze ha dato luogo ad un sistema che può essere considerato come un modello di per sé, tanto che, in una prospettiva comparatistica, non è solo il juicio de amparo a meritare una speciale attenzione, ma anche la combinazione tra le diverse forme di controllo di costituzionalità e l'approccio sincretico che in Messico si è riusciti ad adottare con esiti che meritano di essere rimarcati.

\section{DA TOCQUEVILLE... (IL JUICIO DE AMPARO)}

L'influenza di Alexis de Tocqueville e del suo La democrazia in America ${ }^{4}$ è attestata in maniera incontrovertibile, sia con riguardo alla recezione del principio generale della separazione dei poteri sia, ed in particolare, per ciò che attiene alla elaborazione di forme di scrutinio da parte dei giudici che fossero idonee a proteggere $\mathrm{i}$ diritti individuali senza incidere negativamente sulle prerogative dei pubblici poteri. ${ }^{5}$ La protezione dei diritti doveva infatti essere ottenuta senza che ciò si traducesse nella supremazia dei giudici rispetto al potere politico. Tocqueville aveva descritto il judicial review di marca statunitense precisamente come lo strumento ideale per raggiungere questo bilanciamento, soprattutto in ragione del fatto che in esso le dichiarazioni di incostituzionalità avevano effetti esclusivamente inter partes. Per questo, nel momento in cui si andava ad elaborare una nuova struttura costituzionale, prima nello Yucatan e poi nella Federazione messicana, si trasse ispirazione proprio dalle parole di Tocqueville, il quale venne, infatti, espressamente citato durante i dibattiti che portarono all'introduzione di una nuova via di accesso alle corti, ed in particolare nell'opinione scritta da Mariano Otero avente ad oggetto l'Acta constitutiva y de reformas de 1847. La cosiddetta «Formula Otero», che altro non

3 Si utilizza, in questa sede, una nozione di sistema «accentrato» che diverge rispetto alla definizione che ne ha dato la Corte suprema messicana (cfr. Suprema Corte de Justicia de la Nación, plenum, sentenza 16 aprile 2015, amparo directo en revisión No. 1046/2012). Infra, al par. 5, si cercherà di dar conto delle ragioni per le quali si ritiene di non dover seguire la definizione correntemente in uso in Messico.

4 A. de Tocqueville, De la Démocratie en Amérique, Paris, Pagnerre, 1835-1840.

5 V. Hernández Álvarez, Nartha Maria del Carmen, "Influencia del pensamiento de Alexis de Tocqueville en en juicio de amparo mexicano", in Ferrer Mac-Gregor, Eduardo e Herrera García, Alfonso (eds), El juicio de amparo en el centenario de la Constitución Mexicana de 1917. Pasado, presente y futuro, Mexico, UNAM, Instituto de Investigaciones Jurídicas, 2017, vol. 1, pp. 115-122. 
era se non la traduzione messicana della dichiarazione giudiziale di incostituzionalità resa dai giudici americani, ${ }^{6}$ rappresentava la risposta all'esigenza di garantire una protezione (un amparo) dei diritti nei confronti delle azioni e dei provvedimenti delle pubbliche autorità che fossero lesivi di situazioni giuridiche soggettive (e, di conseguenza, delle corrispondenti previsioni costituzionali), ferma restando l'impossibilità per le corti di esercitare un potere legislativo. ${ }^{7}$

Dal 1841, quando venne introdotto per la prima volta nello Yucatan, e dal 1847, quando venne trasposto a livello federale, il juicio de amparo è divenuto, in sostanza, uno elemento centrale nel sistema messicano di protezione dei diritti. I molteplici sviluppi che il diritto costituzionale ha subito nel corso della storia successiva non hanno inciso su tale centralità, visto che l'idea che il juicio de amparo sia uno strumento per contestare, di fronte ad un giudice, le azioni e gli atti dei pubblici poteri è rimasta inalterata dinanzi alle (pur notevoli) riforme succedutesi.

6 "A esa redacción se le ha llamado fórmula Otero, pero en realidad la afortunada expresión del notable jurista y político jalisciense no significa otra cosa que la adopción de la judicial review norteamericana, divulgada por el clásico libro de Alexis de Tocqueville, La Democracia en América del Norte. En su famoso voto particular, Mariano Otero señaló que se pretendía implantar en nuestro país el sistema estadounidense, y cita varias expresiones que indudablemente toma del escritor francés": così Fix-Zamudio, Héctor, "La declaración general de inconstitucionalidad en Latinoamérica y el juicio de amparo mexicano", Anuario Iberoamericano de Justicia Constitucional, 2002, pp. 87-142, spec. p. 135. Sulla «formula Otero», v. Fernández Segado, Francisco, "El voto particular de don Mariano Otero y el nacimiento del juicio de amparo en México. Nota introductoria sobre los antecedentes des juicio de amparo", Anuario Iberoamericano de Justicia Constitucional, 2000, pp. 579-614; Elías Mussi, Edmundo e Silva Ramírez, Luciano, "La fórmula Otero y la declaratoria general de inconstitucionalidad en el juicio de amparo contra normas", in González Oropeza, Manuel e Ferrer Mac-Gregor, Eduardo (eds), El juicio de amparo. A 160 años de la primera sentencia, Mexico, UNAM, Instituto de Investigaciones Jurídicas, 2011, pp. 27-48.

7 Sull'impatto delle sentenze concernenti questioni di costituzionalità rese prima che la reforma del 2011 mutasse significativamente l'assetto del sistema (cfr. infra, nel testo), v. Fix-Zamudio, Héctor e Ferrer Mac-Gregor, Eduardo, "Las sentencias de los tribunales constitucionales en el ordinamiento mexicano", Anuario Iberoamericano de Justicia Constitucional, 2008, pp. 199-262; Martínez Estrada, Ricardo "Las sentencias de los tribunales constitucionales", in Corzo Sosa, Edgar (ed.), I Congreso Internacional sobre Justicia Constitucional, Mexico, UNAM, Instituto de Investigaciones Jurídicas, 2009, pp. 301-315; Orozco Henríquez, José de Jesús, "Las sentencias de control de la constitucionalidad de normas generales electorales en México", idem, pp. 195-222; Ortiz Mayagoitia, Guillermo Iberio, "Tipología de las sentencias constitucionales que emite la Suprema Corte de Justicia de la Nación”, idem, pp. 223-250. 
Può stupire che, per quel che concerne la struttura del sistema di giustizia costituzionale, il juicio de amparo abbia avuto effetti significativamente diversi rispetto a quelli che si sarebbero potuti immaginare rifacendosi al modello che aveva costituito la fonte di ispirazione. Come noto, il controllo di costituzionalità statunitense è il prototipo dei sistemi diffusi, in quanto consente a qualunque corte di dichiarare che un atto viola la Costituzione. Di contro, il juicio de amparo si è sostanziato in una delimitazione del numero delle corti abilitate a proteggere i diritti nei confronti dell'azione dei pubblici poteri: trattandosi di una procedura giurisdizionale speciale, sin dall'inizio l'amparo è stato concepito come la sola procedura percorribile al fine di ottenere tutela per violazioni costituzionali; così, stante il fatto che la procedura si radica di fronte alle giurisdizioni federali, l'esistenza dell'amparo ha reso impossibile per le corti statali il far valere la Costituzione federale per censurare atti ed azioni del potere al fine di proteggere diritti. In quest'ottica, non è un caso che la Corte suprema messicana definisca la procedura di amparo come l'espressione di un sistema accentrato di giustizia costituzionale: ${ }^{8}$ per quanto la sua introduzione abbia consentito, a svariate corti, di controllare atti ed azioni pubbliche, il suo risultato è stato anche quello di circoscrivere il novero di corti abilitate a svolgere tale scrutinio, con il che può dirsi che, in ultima analisi, l'amparo ha accentrato al livello federale la protezione dei diritti costituzionali.

La distinzione tra le corti federali e le corti statali è sempre stato un elemento fondamentale del sistema basato sull'amparo: l'art. 103, in combinazione con l'art. 107, della Costituzione federale del 1917, nelle sue successive modifiche, anche recenti, concentra inequivocabilmente l'amparo nelle mani delle corti federali.

Il principio secondo cui l'amparo è un rimedio giurisdizionale azionabile solo a livello di corti federali vale per tutti i tipi di amparo. In proposito, giova rammentare che l'idea di offrire uno strumento giurisdizionale per la protezione dei diritti individuali riconosciuti dalla Costituzione contro atti della pubblica autorità si concretizza in diverse modalità, a seconda del tipo di atti che vengano contestati. Un amparo indiretto può essere azionabile contro leggi, trattati internazionali, regolamenti, decreti o altri atti, e più in generale contro qualunque atto dei pubblici poteri che non siano sentenze definitive, che, ad avviso del ricorrente, rappresentino una violazione dei suoi diritti costituzionali; il ricorso deve essere presentato di fronte ad un

\footnotetext{
8 Cfr. la sentenza menzionata supra, nota 3.
} 
giudice distrettuale (Juzgados de Distrito) oppure, quanto si contesta un atto adottato da un giudice distrettuale, di fronte ad un giudice di circuito in composizione monocratica (Tribunal Unitario de Circuito). Al fine di contestare una sentenza definitiva, invece, deve essere presentato un amparo diretto di fronte ad un giudice di circuito in composizione collegiale (Tribunales Colegiados de Circuito).

L'amparo indiretto e l'amparo diretto differiscono anche con riferimento all'impugnazione della decisione di prime cure.

Per l'amparo indiretto, le decisioni rese dal giudice distrettuale o dal giudice monocratico di circuito sono soggette ad impugnazione di fronte ad un giudice collegiale di circuito (amparo indirecto en revisión). L'impugnazione è però presentata direttamente alla Suprema Corte de Justicia de la Nación, d'ufficio o su istanza di parte, quando, in un caso che attiene alla costituzionalità di una legge federale o di un trattato internazionale, non si hanno precedenti, l'esito del giudizio è di eccezionale interesse e si ritiene necessaria una interpretazione del testo costituzionale in quanto di speciale rilevanza per l'ordinamento giuridico nazionale.

Con riguardo all'amparo diretto, invece, la decisione del giudice collegiale di circuito può essere impugnata di fronte alla Corte suprema di giustizia (amparo directo en revisión), ma solo nel caso in cui si debba decidere in ordine ad una questione inerente alla costituzionalità o meno di una regola generale oppure quando si tratti di chiarire l'interpretazione da dare ad una previsione costituzionale o alla formulazione di un diritto umano riconosciuto in un trattato internazionale; ad ogni modo, il caso sottoposto alle giurisdizioni deve richiedere una decisione di speciale rilevanza (come regola generale, dunque, una decisione della Corte suprema di giustizia è richiesta essenzialmente per chiarire la questione giuridica emersa).

È da notare che, in taluni casi eccezionali, la Corte suprema di giustizia, per l'amparo diretto, può esercitare la giurisdizione in unico grado (amparo directo trascendental): ciò si verifica quando la questione è così importante che si impone una decisione della più alta corte del Paese. In queste circostanze, la Corte suprema può avocare a sé la questione, agendo dunque ex officio, oppure può essere destinataria di una richiesta in tal senso da parte del giudice collegiale di circuito o del Procuratore generale della Repubblica. ${ }^{9}$

9 Una sintetica, ma compiuta analisi del juicio de amparo in Messico è consultabile in México, in: Estructura y competencia de las cortes y tribunales supremos de justicia en Iberoamérica, Mexico, Suprema Corte de Justicia de la Nación, 2016, pp. 549-622, spec. p. 567. 
Come si vede, anche se l'amparo è circoscritto all'interno della giurisdizione federale, il numero dei giudici legittimati ad esercitare il sindacato di costituzionalità è lungi dall'essere esiguo. A questo proposito, proprio come in un sistema diffuso, la certezza del diritto rappresenta un problema con cui fare i conti, tanto più che l'ordinamento messicano, non essendo avendo un sistema di common law, non può avvalersi degli strumenti offerti dalla doctrine del precedente vincolante per assicurare la coerenza tra le pronunce giurisdizionali. La questione della certezza del diritto non è del tutto estranea ad un'altra, vale a dire a quella del possibile sovraccarico per le corti, ed in particolare per quella posta al vertice del sistema, che è chiamata a svolgere un ruolo di chiusura per tutta la giurisdizione federale.

La strada che, al riguardo, si è percorsa in Messico per dare efficienza al sistema è certamente originale, poiché innesta alcune soluzioni ispirate al common law all'interno di una struttura di civil law. Due aspetti del sistema meritano una particolare attenzione.

Il primo aspetto attiene ai meccanismi di impugnazione che si sono sommariamente descritti, i quali mostrano chiaramente che la Corte suprema di giustizia dovrebbe, in linea di principio, essere adita solo per casi particolarmente importanti. Sebbene i due sistemi funzionino in modo molto diverso, gli effetti della selezione fatta in Messico sulla base della rilevanza della questione da decidere può richiamare, da un punto di vista teorico, gli effetti del writ of certiorari per la Corte suprema federale statunitense. In concreto, tuttavia, emerge tra i due una chiara differenza, poiché la Corte suprema messicana non ha un reale potere di selezione dei casi, ed è dunque costretta a fronteggiare un carico di lavoro che è considerevolmente maggiore di quello dell'omologo statunitense. ${ }^{10}$ La comparazione è interessante anche con riferimento alla nozione di «impugnazione», dal momento che la selezione dei casi operata in relazione all'amparo messicano è basata sull'interesse generale, il che la avvicina all'idea che conforma l'impugnazione nei sistemi di common law, mentre la regola generale dei sistemi di civil

10 Prendendo in considerazione il periodo compreso tra il 2013 ed il 2016, il numero di casi pervenuti annualmente è stato compreso tra i 6.000 e gli 8.000. I giudizi originati da un amparo indiretto sono quelli che più contribuiscono al sovraccarico per la Corte, visto che il loro numero eccede sempre i 2.000 per anno e, sovente, anche i 3.000. Fonte: Suprema Corte de Justicia de la Nación, Secretariá de Acuerdos - Estadística Judicial, Estadística mensual. Mayo 2017, consultabile on line alla pagina http://www.scjn.gob. $\mathrm{mx} /$ sites/default/files/pagina/documentos/2017-06/SGAEEM0517.pdf (ultimo accesso: 12 agosto 2018). 
law, pur con alcune notevoli eccezioni, ${ }^{11}$ è che il diritto all'impugnazione si lega essenzialmente all'interesse della parte.

Il secondo aspetto da prendere in considerazione concerne gli effetti delle pronunce che decidono ricorsi di amparo. La «Formula Otero», come si è visto, era collegata all'obiettivo di assicurare che le sentenze avessero soltanto effetti inter partes. Ora, poiché il sistema messicano rientra nel novero di quelli di civil law, non c'è ovviamente modo di imporre la regola dello stare decisis. Tuttavia, il principio della efficacia meramente inter partes delle pronunce è stata molto presto assoggettata ad un ripensamento, alla luce del riconoscimento di effetti vincolanti alla c.d. jurisprudencia, vale a dire all'orientamento giurisprudenziale che si sostanzia nella ripetizione della stessa ratio in pronunce rese da corti superiori, e cioè dalla Corte suprema di giustizia o da giudici collegiali di circuito. ${ }^{12}$ Più precisamente, la Corte suprema stabilisce una jurisprudencia quando conferma la stessa ratio in cinque successive decisioni rese in diverse sessioni. Per attribuire efficacia vincolante ad una ratio ripetuta, le pronunce debbono essere adottate dal plenum con una maggioranza di almeno otto voti su undici oppure da una sezione della Corte con una maggioranza di almeno quattro voti su cinque. Per quanto attiene ai giudici collegiali di circuito, le pronunce richiedono l'unanimità del collegio di tre giudici.

Questo sistema venne introdotto dalla Ley de Amparo del 1882, proprio per garantire la certezza del diritto in un sistema caratterizzato dall'assenza di una dottrina del precedente. Da allora, si è sviluppato un ampio dibattito sulla portata degli effetti vincolanti della jurisprudencia. Due

\footnotetext{
11 Ad esempio, il diritto ad un giudizio di merito da parte dell'organo di giustizia costituzionale su un ricorso diretto individuale è generalmente soggetto a considerazioni riguardanti l'interesse generale del caso e di una possibile decisione su di esso. A tal proposito, le innovazioni apportate dalla legge organica n. 6 del 2007 al recurso de amparo di fronte al Tribunale costituzionale spagnolo sono esempi emblematici, poiché il cambiamento più significativo è consistito proprio nel rafforzamento del peso dell'interesse generale nella selezione dei casi.

12 Per una descrizione delle modalità attraverso cui una jurisprudencia viene a formarsi, v. López Benítez, Lilia Mónica, "Sistemas de jurisprudencia en el juicio de amparo", in Ferrer Mac-Gregor, Eduardo e Herrera García, Alfonso (eds), El juicio de amparo en el centenario de la Constitución mexicana de 1917, op cit., vol. 2, pp. 535-549; v. anche, con particolare riguardo ai rapporti tra la jurisprudencia e la dichiarazione di incostituzionalità, Coello Garcés, Clicerio e Herrera García, Alfonso, “Jurisprudencia por reiteración y declaratoria general de inconstitucionalidad de leyes en el juicio de amparo", idem, vol. 2, pp. 513-533.
} 
tesi principali sono state avanzate: l'una definisce la jurisprudencia come una fonte formale del diritto, come tale vincolante per tutte le autorità pubbliche, l'altra limita gli effetti della jurisprudencia all'interpretazione delle disposizioni normative, con il risultato che i suoi effetti vincolanti si dispiegherebbero soltanto all'interno della giurisdizione, senza incidere sulle altre autorità.

Qualche anno fa, il juicio de amparo ha conosciuto importanti riforme, che hanno avuto ad oggetto anche la «formula Otero». In effetti, la riforma costituzionale del 6 giugno 2011, attuata con la Nueva Ley de Amparo del 2 aprile 2013, ha introdotto, tra l'altro, una nuova procedura volta a consentire alla Corte suprema di giustizia di dichiarare, nell'ambito di un procedimento di amparo, l'incostituzionalità di previsioni normative..$^{13}$

Ai termini dell'art. 107, par. secondo, della Costituzione, come risultante dalle modifiche, la dichiarazione di incostituzionalità ha effetti erga omnes, quindi espunge dall'ordinamento giuridico le previsioni di cui si constata la incompatibilità con la Costituzione. Questo nuovo strumento per reagire contro gli atti delle pubbliche autorità è stato concepito per rafforzare la superiorità della Costituzione, ma anche per ridurre il carico di lavoro imposto alle corti, oltre che per rafforzare l'eguaglianza e, soprattutto, per migliorare, in generale, la protezione dei diritti, grazie all'impatto di una dichiarazione che dispiega i propri effetti su tutti i consociati, e non solo sulle parti in giudizio. ${ }^{14}$

In concreto, se la Corte suprema di giustizia, nel decidere ricorsi in sede di amparo indiretto, constata - in due distinti procedimenti- che una disposizione normativa (in qualunque settore tranne che nel diritto tributario) è incostituzionale, il presidente della Sezione che ha reso la seconda constatazione o il presidente della Corte, se a pronunciarsi è stato il plenum, informa l'autorità cui l'atto è imputabile della decisione resa dalla Corte.

13 Gli effetti del nuovo assetto normativo relativo al juicio de amparo sono presi in esame da Carranco Zúñiga, Joel, "Diagnóstico del juicio de amparo a cuatro años de vigencia de la nueva ley", in Ferrer Mac-Gregor, Eduardo e Herrera García, Alfonso (eds.), El juicio de amparo en el centenario de la Constitución mexicana de 1917, op. cit., vol. 1, pp. 167-186; Ojeda Bohórquez, Ricardo, "Actualidad del juicio de amparo a 100 años de la Constitución mexicana de 1917”, idem, vol. 1, pp. 201-214.

14 Sul tema, v. Figueroa Mejía, Giovanni Azael, "Efectos de las sentencias de amparo: modificación parcial del principio de relatividad a través de la declaratoria general de inconstitucionalidad", in Ferrer Mac-Gregor, Eduardo e Herrera García, Alfonso (eds), El juicio de amparo en el centenario de la Constitución mexicana de 1917, op. cit., vol. 2, pp. 393-407. 
Analogamente, l'autorità è informata quando, in sede di amparo diretto, la Corte suprema di giustizia stabilisce una jurisprudencia secondo la quale una disposizione (in qualunque settore tranne che nel diritto tributario) è incostituzionale.

Una volta che all'autorità interessata sia stata notificata la decisione, iniziano a decorrere i novanta giorni che le vengono riservati per modificare o abrogare la disposizione considerata incostituzionale. Se l'autorità non elimina il vizio di costituzionalità, la Corte suprema, in seduta plenaria, pronuncia la dichiarazione di incostituzionalità. Perché la dichiarazione sia validamente resa, è richiesta una maggioranza di almeno otto voti.

Con questa nuova procedura, la Corte suprema di giustizia è stata dotata di una attribuzione tipica di un sistema accentrato di giustizia costituzionale, con la conseguenza che il juicio de amparo, nel suo complesso, ha visto incrementare le sue peculiarità: pur essendo una procedura diretta specificamente al controllo degli atti dei pubblici poteri a beneficio dei ricorrenti, non trascura (più) una diversa prospettiva e diversi obiettivi, come, in special modo, quello di garantire la superiorità della Costituzione in termini generali ed astratti.

Questa evoluzione potrebbe suggerire l'esistenza di un percorso verso il recepimento del modello europeo di giustizia costituzionale, o meglio verso l'avvicinamento ai sistemi misti basati sul modello europeo, in cui il controllo concreto di costituzionalità è associato ad una possibile dichiarazione con effetti erga omnes dell'incompatibilità delle disposizioni scrutinate con la Costituzione. A ben vedere, tuttavia, i mutamenti che sono intervenuti nel sistema messicano sono molto più complessi: nei paragrafi seguenti si cercherà di darne conto.

\section{III. ... A MARSHALL (L'INTRODUZIONE DI UN CONTROLLO DI COSTITUZIONALITÀ DIFFUSO)}

Pur non essendo un sistema accentrato (salvo quanto si è accennato e si dirà in ordine alla qualificazione data dalla Corte suprema), il sistema basato esclusivamente sul juicio de amparo è stato caratterizzato da una difficoltà che colpisce praticamente tutti i sistemi accentrati, e cioè l'esistenza di aree dell'ordinamento prive di protezione in ragione, per un verso, dell'impossibilità per i giudici comuni di disapplicare atti e disposizioni incompatibili con la Costituzione e, per l'altro, dell'impossibilità 
di ottenere adeguati rimedi giurisdizionali per qualunque tipo di lesione lamentata.

Questa duplice inefficienza ha caratterizzato il sistema messicano per un lungo periodo, specie con riferimento ai giudici statali, i quali non hanno avuto possibilità di svolgere un sindacato sulla legislazione sino a quando il panorama giuridico non è cambiato in maniera significativa. Ciò si è verificato nel 2011, per effetto del combinarsi di una importante riforma costituzionale e di una altrettanto importante risoluzione della Corte suprema.

La revisione costituzionale del 10 giugno 2011 ha avuto un impatto considerevole sulla posizione del diritto internazionale in seno all'ordinamento giuridico messicano. L'art. 1, par. primo, della Costituzione, come emendato, tutela esplicitamente i diritti individuali per come riconosciuti dalla Costituzione e dai trattati internazionali firmati dal Messico, ed impedisce qualunque limitazione a questi diritti, «salvo en los casos y bajo las condiciones que esta Constitución establece».

Il rango dei trattati internazionali nell'ordinamento interno è ulteriormente chiarito nell'art. 1, par. secondo, che così recita: «[l] as normas relativas a los derechos humanos se interpretarán de conformidad con esta Constitución y con los tratados internacionales de la materia favoreciendo en todo tiempo a las personas la protección más amplia».

La protezione dei diritti è quindi basata sia sulla Costituzione che sui trattati internazionali, e se la prima prevale quando si tratta di limitazioni che vengono imposte ai diritti (art. 1, par. primo), in riferimento all'interpretazione non si dà, invece, una gerarchia rigida, poiché il criterio guida è quello di estendere la protezione dei diritti (par. secondo). ${ }^{15}$

Il rango quasi identico che hanno la Costituzione ed i trattati internazionali è ulteriormente confermato all'art. 133, come modificato, che precisa i rapporti tra diritto federale e diritto statale stabilendo che la "Constitución, las leyes del Congreso de la Unión que emanen de ella y todos los tratados que estén de acuerdo con la misma, celebrados y que se celebren por el Presidente de la República, con aprobación del Senado, serán la Ley Suprema de toda la Unión». Conseguentemente, «[l]os jueces de cada entidad

15 Con riferimento all'interpretazione della Costituzione e dei trattati internazionali alla luce della prevalenza che deve essere accordata alla protezione dei diritti, v. Ruiz Legazpi, Ana, "La cláusula de simpatía con los derechos en México: la aplicación de los tratados y la jurisprudencia international sobre los derechos humanos tras la reforma constitucional de 2011 (en especial, Convención y Corte Interamericana de Derechos Humanos)", Anuario Iberoamericano de Justicia Constitucional, 2013, pp. 287-309. 
federativa se arreglarán a dicha Constitución, leyes y tratados, a pesar de las disposiciones en contrario que pueda haber en las Constituciones o leyes de las entidades federativas».

L'impianto normativo che si articola sull'insieme di queste previsioni è stato alla base di una fondamentale risoluzione resa dalla Corte suprema di giustizia, ${ }^{16}$ con cui si è stabilito il principio secondo il quale

...[s]i bien los jueces no pueden hacer una declaración general sobre la invalidez o expulsar del orden jurídico las normas que consideren contrarias a los derechos humanos contenidos en la Constitución y en los tratados..., sí están obligados a dejar de aplicar estas normas inferiores dando preferencia a los contenidos de la Constitución y de los tratados en esta materia. ${ }^{17}$

La Corte suprema ha così posto le basi per integrare il sistema messicano di giustizia costituzionale, arricchendolo di un controllo di costituzionalità e di convenzionalità. ${ }^{18}$ In effetti, con la risoluzione del 2011, in Messico, qualunque giudice, nell'esercizio delle sue attribuzioni, è legittimato a verificare se una disposizione normativa è compatibile con la Costituzione o con i trattati internazionali vincolanti per l'Unione messicana e, in caso di accertata incompatibilità, lo stesso giudice può disapplicare la disposizione al fine di proteggere un diritto riconosciuto dalla Costituzione o dai trattati internazionali. Alla luce dell'interpretazione fatta propria dalla Corte suprema, l'art. 133 della Costituzione, nella sua versione più recente, ha permes-

16 Expediente Varios No. 912/2010, "Rosendo Radilla Pacheco Case", risoluzione emanata il 14 luglio 2011.

17 Id., par. 29

18 Per quel che concerne l'impatto della risoluzione della Corte suprema e la conseguente introduzione di un sistema diffuso di giustizia costituzionale, v. Herrera García, Alfonso, "Jurisprudencia constitucional de la Suprema Corte de Justicia de México en 2011", Anuario Iberoamericano de Justicia Constitucional, 2012, pp. 443-474; Hallivis Pelayo, Manuel Luciano, "Control difuso de la convencionalidad", in Ferrer Mac-Gregor, Eduardo e Herrera García, Alfonso (eds), El juicio de amparo en el centenario de la Constitución mexicana de 1917, op. cit., vol. 2, pp. 287-297; A. Herrera García, Alfonso, "El control difuso de regularidad de la leyes en el juicio de amparo directo: complejidades conceptuales y frente al sistema interamericano de derechos humanos", idem, vol. 2, pp. 299-316; Pardo Rebolledo, Jorge Mario, López Andrade, Guillermo Pablo e Silva Díaz, Ricardo Antonio, "Control de convencionalidad y bloque de regularidad constitucional en el juicio de amparo", idem, vol. 2, pp. 317-333. In lingua inglese, v. Narváez Medécigo, Alfredo, "Constitutional Review in Mexico: A Best of All Worlds Solutions?", idem, Rule of Law and Fundamental Rights, Berlin, Springer, 2016, pp. 213-258. 
so ai giudici statali di svolgere il controllo di costituzionalità, attribuendo loro, in tal modo, un potere che non era mai stato riconosciuto ${ }^{19}$ - e che sarebbe stato esercitato quasi immediatamente-,${ }^{20}$ un potere destinato a ridurre drasticamente il divario tradizionale intercorrente tra le giurisdizioni federali e le giurisdizioni statali. ${ }^{21}$

Con l'attribuzione a tutti i giudici messicani del potere di controllare la costituzionalità (e la convenzionalità) del diritto nazionale, la protezione dei diritti ha potuto beneficiare di una consistente riduzione delle zone grigie sprovviste di tutela giurisdizionale. Una significativa difficoltà, tuttavia, residuava: il riferimento è all'incertezza dell'attribuzione anche ai giudici federali di un potere generalizzato di controllo della costituzionalità degli atti. Più in particolare, l'esistenza del juicio de amparo poteva rappresentare, paradossalmente, un limite alla giurisdizione federale, nel senso che si poteva ritenere che i giudici federali fossero in grado di operare il controllo di costituzionalità soltanto in sede di amparo, e dunque che il loro potere di sindacato fosse soggetto alla tipologia di azione radicata da parte del ricor-

19 Ancora nel 2010, il fatto che ai giudici statali fosse impedito qualunque sindacato di costituzionalità sulle leggi era considerato «more a product of judicial interpretation at the federal level rather than an explicit institutional arrangement set in the Constitution»: cfr. García Sarubbi, David, "Federalism and Constitutional Judicial Review in Mexico and the United States: A Normative Assessment of Two Different Jurisdictional Schemes", Mexican Law Review, 2011, No. 1, pp. 35-58, spec. p. 41.

20 Il primo caso in cui un giudice statale ha disapplicato una disposizione legislativa statale in conseguenza della sua incompatibilità con i diritti umani risale a poche settimane dopo la emanazione della risoluzione della Corte suprema: in effetti, la sentenza nel c.d. caso Nuevo León case è stata resa 1'8 agosto 2011. Per l'analisi dei collegamenti tra la risoluzione della Corte suprema e la sentenza della giurisdizione statale, v. Narváez Medécigo, Alfredo, "Enforcement of Fundamental Rights by Lower Courts: Towards a Coherent System of Constitutional Review in Mexico", Mexican Law Review, 2013, no. 1, pp. 3-44, spec. p. 36.

21 Focalizzando l'attenzione sui livelli federale ed internazionale, la riforma costituzionale, ovviamente, non ha potuto prendere in considerazione il tema della protezione delle costituzioni statali. Questa tematica, che non sarà oggetto di analisi nel presente scritto, ha peraltro conosciuto significative innovazioni dall'inizio di questo secolo, innovazioni tese ad introdurre, in vari stati della Unione messicana, una «giustizia costituzionale locale»: cfr. Casarín León, Manlio Fabio, "Justicia constitucional local: retos y perspectivas", in Corzo Sosa, Edgar (ed.), I Congreso Internacional sobre Justicia Constitucional, op. cit., pp. 375-398; Astudillo Reyes, César Iván, "La justicia constitucional local en México: presupuestos, sistemas y problemas", idem, pp. 325-374; Carnota, Walter Fabián, "La justicia constitucional en el constitucionalismo subnacional mexicano", Anuario Iberoamericano de Justicia Constitucional, 2016, pp. 69-84. 
rente nel giudizio. Altrimenti detto, a seguire la tesi restrittiva, in assenza di una specifica censura della costituzionalità di una certa disposizione, il giudice federale si sarebbe trovato nell'obbligo di applicarla, indipendentemente dalla sua eventuale incompatibilità con la Costituzione.

L'incertezza a questo riguardo è stata rimossa, alla fine, da una importante sentenza resa dalla Corte suprema di giustizia nel 2015, nella quale si è affermato incontrovertibilmente che il controllo di costituzionalità ex officio è un obbligo che si impone a qualunque giudice, in qualunque caso, con il risultato che non possono più darsi limitazioni derivanti dal tipo di azione promossa dal ricorrente e/o dal tipo di giurisdizione esercitata dal giudice. ${ }^{22}$

Grazie a queste recenti riforme costituzionali ed all'interpretazione seguita dalla Corte suprema, si è instaurato un sistema propriamente diffuso, dal quale è uscita sensibilmente rafforzata la superiorità della Costituzione. ${ }^{23} \mathrm{Il}$ compromesso raggiunto a metà dell'Ottocento con la limitazione del controllo giurisdizionale di costituzionalità attraverso il juicio de amparo ha lasciato spazio, all'inizio del XXI secolo, ad una chiara opzione in favore di un trapianto dell'argomento del Chief Justice Marshall che si pone a fondamento del modello nordamericano di giustizia costituzionale. ${ }^{24}$ Non senza un qualche paradosso, gli Stati Uniti sono stati a lungo una fonte rilevante di ispirazione per la giustizia costituzionale messicana, ma questa fonte non ha mai raggiunto un livello tale da produrre una vera e propria imitazione; soltanto in tempi recenti il modello statunitense è stato finalmente adottato, proprio quando il modello europeo sembrava essere divenuto il punto di riferimento fondamentale per una rivisitazione del sistema messicano. ${ }^{25}$ L'ispirazione «europea» è stata chiaramente attestata

22 Sentenza 16 aprile 2015, No. 1046/2012 (v. supra, nota 3).

23 «Así, el Sistema jurídico mexicano inició una nueva etapa con la expresa constitucionalización de los derechos humanos, lo cual impacta en la tarea de todas las autoridades publicas del país, pues les impone obligaciones concretas con el propósito de alcanzar el pleno respeto y garantía de tales derechos, principalmente las obligaciones interpretativas de los juzgadores»: Góngora Pimentel, Genaro David, "El juicio de amparo y el control difuso de constitucionalidad y convencionalidad", in Ferrer Mac-Gregor, Eduardo e Herrera García, Alfonso (eds), El juicio de amparo en el centenario de la Constitución mexicana de 1917, op. cit., vol. 2, pp. 277-286, spec. p. 278.

24 Il riferimento va, ovviamente, alla opinion del Chief Justice Marshall nel caso Marbury v. Madison, 5 U.S. 137 (1803).

25 Sulle successive ondate di influenze che hanno inciso e contribuito a strutturare il sistema messicano di giustizia costituzionale, v. Narváez Medécigo, Alfredo, Enforcement of Fundamental Rights by Lower Courts, cit., pp. 16-31. 
dall'introduzione di una declaratoria di incostituzionalità con effetti erga omnes nel procedimento di amparo. Tuttavia, non si è trattato né del primo né del più chiaro esempio di influenza proveniente dall'altra sponda dell'Oceano Atlantico. Il paragrafo seguente tratterà, non a caso, di altri caratteri del sistema messicano che richiamano il modello accentrato di controllo di costituzionalità.

\section{IV. ...PASSANDO PER KELSEN (L'ESISTENZA DI UN CONTROLLO ASTRATTO DI COSTITUZIONALITÀ)}

Al momento di redigere la Costituzione del 1917, il juicio de amparo è stato concepito come la pietra miliare del sistema di giustizia costituzionale messicano; ciò nondimeno, il costituente ha lasciato spazio anche ad un'altra forma di controllo. Il riferimento va alla competenza attribuita alla Corte suprema di giustizia di decidere le controversie costituzionali instaurate dalle Camere del Parlamento, dall'Esecutivo federale o dai governatori statali. Nella prassi, questa competenza è stata a lungo esercitata soltanto in maniera episodica: in quasi otto decenni, si è avuta una dozzina di controversie costituzionali, la maggior parte delle quali, tra l'altro, neppure è stata decisa nel merito da parte della Corte suprema. Sulla scorta di questo rilievo, rappresentava una approssimazione assolutamente tollerabile l'identificare pianamente il sistema di giustizia costituzionale messicano con il funzionamento del procedimento di amparo, senza dover in alcun modo prendere in considerazione altre forme di controllo.

Con la riforma del 1994, entrata in vigore il 1o. gennaio 1995, è intervenuto un cambiamento di non poco momento, consistente nell'ampliamento degli ambiti nei quali la Corte suprema esercita una giurisdizione costituzionale: da un lato, il potere di promuovere controversie costituzionali è stato riconosciuto anche ad altri soggetti; dall'altro, è stata introdotta una nuova forma di ricorso.

Con riferimento alle controversie costituzionali, vari altri soggetti si sono visti riconosciuta la legittimazione ad adire la Corte suprema: oltre alle Camere del Parlamento, all'Esecutivo federale ed ai governatori statali, oggi le controversie costituzionali possono essere instaurate dai municipi, dai rappresentanti del difensore civico e dai vertici dei partiti politici. In concreto, questa estensione dell'elenco di possibili ricorrenti 
si è tradotta in una crescita esponenziale del numero di decisioni rese dalla Corte suprema. ${ }^{26}$

Si è, in sostanza, consolidata una prassi in base alla quale, quando una pubblica autorità emana un atto normativo o un provvedimento generale (come una legge, un regolamento o un decreto), che incide sulla sfera di attribuzioni di un'altra autorità, la Corte suprema viene adita perché risolva la controversia. ${ }^{27}$ Quando il caso giunge di fronte alla Corte, lo scrutinio da essa compiuto può estendersi a qualunque tipo di violazione della Costituzione, con il che le controversie costituzionali possono essere agevolmente assimilate ad un vero e proprio controllo di costituzionalità. ${ }^{28}$ In effetti, se la Corte conclude nel senso che l'autorità convenuta ha adottato una previsione generale (come ad esempio una disposizione normativa) esercitando poteri che erano propri di un'altra autorità o di un altro livello di governo, la previsione viene inficiata ed espunta dall'ordinamento giuridico. Perché ciò accada, è peraltro necessario che la Corte suprema di giustizia, in sessione plenaria, adotti la relativa risoluzione con una maggioranza di almeno otto voti su undici. Se tale maggioranza non viene raggiunta, il ricorso che ha dato luogo alla controversia costituzionale è respinto.

La riforma costituzionale del 1994, inoltre, ha attribuito alla Corte suprema giurisdizione sulle acciones de inconstitucionalidad, conferendo così alla Corte il potere di controllare la legislazione in una forma astratta che richiama nitidamente i procedimenti che si svolgono di fronte alle corti costituzionali del continente europeo. ${ }^{29}$

26 Ad esempio, dal 2009, il numero di controversie costituzionali pervenute annualmente ha sempre ecceduto quota 100, tranne che nel 2012 (quando il numero è stato inferiore: 84); nel 2010 e nel 2013 il totale ha superato quota 200 (con, rispettivamente, 206 e 203). Fonte: Suprema Corte de Justicia de la Nación, Secretariá de Acuerdos - Estadística Judicial, Estadística mensual. Mayo 2017, op. cit.

27 Per quel che concerne l'impatto della riforma costituzionale sullo sviluppo del contenzioso relativo alle controversie costituzionali, v. Silva Meza, Juan, "La Cour suprême de justice de la Nation du Mexique. La Révolution mexicaine des droits de 1'homme", Nouveaux Cahiers du Conseil constitutionnel, 2013, No. 39, pp. 317-326, spec. p. 320.

28 Gli effetti della riforma costituzionale sulla giurisprudenza della Corte suprema sono analizzati da Orozco Henriquez, José de Jesús, "La Suprema Corte de Justicia de la Nación a partir de 1995 y el nuevo orden constitucional", Anuario Iberoamericano de Justicia Constitucional, 2006, pp. 281-302.

29 Recentemente, il numero di azioni promosse annualmente ha oscillato tra le 10 (nel 2010) e le 59 (nel 2016), ma la soglia dei 20 casi è stata comunque raggiunta quasi ogni anno. Fonte: Suprema Corte de Justicia de la Nación, Secretariá de Acuerdos - Estadística Judicial, Estadística mensual. Mayo 2017, op. cit. 
Legittimati ad adire la Corte supreme sono le minoranze parlamentari (un terzo dei membri di una delle due Camera del Parlamento federale ovvero di uno dei trentadue parlamenti statali), i governatori, i sindaci, il Procuratore generale della Repubblica ed il Presidente della Commissione per i diritti umani.

L'azione di incostituzionalità è lo strumento atto a far controllare la costituzionalità di leggi che concentra il sindacato esclusivamente presso la Corte suprema di giustizia. Il ricorso deve essere presentato entro trenta giorni dalla pubblicazione della previsione contestata. ${ }^{30}$

Se la Corte suprema dichiara la disposizione incompatibile con la Costituzione, la disposizione non può più essere applicata ed è espunta dall'ordinamento giuridico. In altri termini, la dichiarazione di incostituzionalità ha effetti generali. Anche in questo caso, è necessaria l'approvazione da parte del plenum, con una maggioranza di almeno otto giudici su undici.

L'azione di incostituzionalità non può in alcun modo dirsi sostitutiva del procedimento di amparo, in quanto gli obiettivi delle due procedure sono diversi: l'amparo mira alla protezione dei diritti, mentre l'azione di incostituzionalità è diretta principalmente a mantenere una coerenza all'interno del sistema delle fonti del diritto. Quest'ultimo obiettivo è, come ovvio, strettamente legato sia alla garanzia della Costituzione che alla protezione dei diritti; tuttavia, il raggiungimento di queste finalità ulteriori appare consequenziale. Come dire che la nuova attribuzione della Corte suprema di giustizia integra i requisiti identificati da Hans Kelsen come caratterizzanti delle corti costituzionali; ${ }^{31}$ questi stessi requisiti, del resto, connotano, anche oggi, il modello europeo di giustizia costituzionale, o meglio sono generalmente parte dell'arsenale di attribuzioni su cui possono contare le corti costituzionali sul continente europeo. A ben vedere, infatti, il modello europeo è in larga misura un ibrido, in cui il controllo astratto di matrice kelseniana è una delle vie d'accesso alla giurisdizione costituzionale, ma in cui sono state introdotte anche forme di controllo concreto, specie attraverso meccanismi di ricorso diretto individuale o di

30 «Pour la première fois, la possibilité s'est ouverte pour les perdants du débat parlementaire d'obtenir tout de même l'annulation juridictionnelle d'une loi pour un défaut juridique quelconque»: Silva Meza, Juan, La Cour suprême de justice de la Nation du Mexique, op. cit., p. 321.

31 Il testo di riferimento al riguardo è, ovviamente, Kelsen, Hans, "La Garantie juridictionnelle de la constitution (la Justice constitutionnelle)", Revue du droit public et de la science politique, 1928, pp. 197-257. 
instaurazione di un sindacato di tipo incidentale promosso da giudici nel corso di un giudizio.

In questo contesto, il sistema messicano, per quanto sia un esempio di ibridazione, non presenta le stesse caratteristiche della maggior parte dei sistemi europei di giustizia costituzionale: in Messico, la coesistenza di procedimenti diversi per il controllo di costituzionalità non è il risultato di una scelta di un determinato modello (vale a dire quello proposto da Kelsen) con alcune deroghe (segnatamente, il controllo concreto); piuttosto, il carattere ibrido discende dalla confluenza di diverse ispirazioni. Alla luce di questo, quello messicano può dirsi davvero un sistema misto, probabilmente in termini più pregnanti rispetto a quelli che solitamente vengono definiti tali dalla scienza comparatistica.

\section{L'IBRIDAZIONE COME RISULTATO DI UN SINCRETISMO: LA VIA MESSICANA AL CONTROLLO DI COSTITUZIONALITÀ}

Nella pronuncia del 2015 che si è in precedenza ricordata, la Corte suprema ha distinto tra sistemi accentrati e sistemi diffusi di giustizia costituzionale, definendo il ricorso di amparo come espressione di sistemi del primo tipo. ${ }^{32}$ Un osservatore europeo resta piuttosto sorpreso da una tale qualificazione: in Europa, infatti, il concetto di sistema accentrato è generalmente associato all'esistenza di una corte specializzata che abbia il monopolio del controllo di costituzionalità. Per questa ragione, un punto di vista esterno condurrebbe a privilegiare una collocazione del juicio de amparo in posizione intermedia tra il controllo diffuso, nelle forme delineate dalla Corte suprema di giustizia nel 2011, ed il controllo propriamente accentrato, riconducibile alle azioni di incostituzionalità ed alle controversie costituzionali.

Ne discende che il sistema messicano di giustizia costituzionale può essere definito come il risultato della somma di tre diversi tipi di controllo di costituzionalità, tutti derivanti da modelli differenti: il modello «autoctono», il modello nordamericano ed il modello europeo.

In questa coesistenza di diverse, autonome forme di controllo risiede la vera peculiarità del sistema messicano, una peculiarità che distingue chiara-

32 Per l'analisi della sentenza, v. anche Herrera García, Alfonso, “Jurisprudencia constitucional de la Suprema Corte de Justicia de México en 2015", Anuario Iberoamericano de Justicia Constitucional, 2016, pp. 493-523. 
mente il Messico da altri sistemi tradizionalmente annoverati tra quelli misti, come quello portoghese o quello greco: in entrambi, infatti, la diffusione del controllo di costituzionalità delle leggi, che consente ai giudici comuni di disapplicare disposizioni incompatibili con la Costituzione, è temperata dal monopolio riconosciuto al Tribunale costituzionale (in Portogallo) o ad una speciale corte suprema (in Grecia) di rendere dichiarazioni di incostituzionalità aventi effetti generali.

Una comparazione più efficace può semmai essere condotta con il sistema brasiliano, nel quale il sindacato diffuso coesiste con una procedura autonoma di matrice accentrata, senza che tra le due procedure esistano punti di intersezione. Inoltre, in Brasile, come in Messico, la giustizia costituzionale è basata essenzialmente sull'attività della Corte suprema. Il Messico, in ogni caso, mantiene una sua peculiarità, non solo in virtù della tripartizione delle ispirazioni, ma anche in conseguenza delle sue radici storiche: la protezione dei diritti costituzionali non deriva da un controllo di costituzionalità diffuso, dal momento che il sistema è stato edificato sull'assunto esplicitato che si dovesse limitare la «diffusione» del sistema.

In definitiva, l'ordinamento messicano sta quindi sperimentando una ricerca di equilibrio tra diverse forme di controllo di costituzionalità. Un equilibrio che ben può dirsi unico nel panorama comparatistico, in quanto l'esistenza di una forma intermedia come quella del juicio de amparo rende ancor più impellente una chiara definizione della portata di ciascuna tipologia di controllo e dei rispettivi limiti. Confrontando quello messicano con altri sistemi, può dirsi che la sua complessità non deriva tanto dal numero di attribuzioni che consentono ai giudici (e/o alla suprema corte) di controllare la costituzionalità di norme e provvedimenti: a questo proposito, il sistema messicano appare tutto sommato piuttosto semplice e lineare. La complessità deriva, piuttosto, dalla divergenza delle forme che consentono di radicare questioni di costituzionalità $\mathrm{o}$, in altri termini, dalle fonti di ispirazione che sono state implementate nel corso del tempo. Sebbene tale coesistenza possa creare conflitti e problemi per il funzionamento del sistema, pare di poter dire, comunque, che in una prospettiva di medio-lungo termine, la ricchezza di strumenti che sono a disposizione del potere giudiziario messicano rappresenta un patrimonio da preservare e da fare ulteriormente sviluppare per rafforzare la protezione della Costituzione e, con essa, dei diritti individuali.

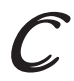

